\title{
RNA-Binding Protein IGF2BP1 Enhances mRNA Stability and Translation Efficiency of INHBA to Promote the Invasion and Migration of Esophageal Squamous Cancer Cells
}

\section{Juan-Juan Wang}

Chinese Academy of Medical Sciences \& Peking Union Medical College

\section{Ding-Xiong Chen}

Chinese Academy of Medical Sciences \& Peking Union Medical College

\section{Yu Zhang}

Chinese Academy of Medical Sciences \& Peking Union Medical College Xin Xu

Chinese Academy of Medical Sciences \& Peking Union Medical College

\section{Yan Cai}

Chinese Academy of Medical Sciences \& Peking Union Medical College Jia-Jie Hao

Chinese Academy of Medical Sciences \& Peking Union Medical College

Ming-Rong Wang ( $\triangle$ wangmr2015@126.com )

Chinese Academy of Medical Sciences \& Peking Union Medical College

\section{Research}

Keywords: esophageal squamous cell carcinoma, metastasis, RNA binding protein, IGF2BP1, INHBA

Posted Date: July 7th, 2021

DOI: https://doi.org/10.21203/rs.3.rs-673905/v1

License: (c) (1) This work is licensed under a Creative Commons Attribution 4.0 International License. Read Full License 


\section{Abstract}

\section{Background}

Metastasis are mainly responsible for the death of patients with advanced esophageal squamous cell carcinoma (ESCC). At present, there is no targeted drug for the treatment of ESCC in clinic practice. The present study aims to investigate the roles and implication of IGF2BP1 overexpression in ESCC.

Methods

IGF2BP1 protein expression was assessed by immunohistochemistry (IHC), and the mRNA abundance of IGF2BP1 and INHBA were analyzed with TCGA datasets and by RNA in situ hybridization (RISH). Cell viability, migration, invasion and in vivo metastasis assays were performed to explore the roles of IGF2BP1 in ESCC. RNA immunoprecipitation sequencing (RIP-seq) and mass spectrometry were applied to identify the targets and interacting proteins of IGF2BP1, respectively. RIP-PCR, RNA-pulldown, immunofluorescence (IF), gene specific $\mathrm{m}^{6} \mathrm{~A} P C R$ and RNA stability assay were used to uncover the molecular mechanism of IGF2BP1 dysregulation. The methylation level of IGF2BP1 promoter region was detected by MSP-PCR. BTYNB, a small molecular inhibitor which could block the binding of IGF2BP1 to CMyc mRNA, was evaluated for the inhibition effect on the malignant phenotypes of ESCC cells.

\section{Results}

IGF2BP1 overexpression was detected in ESCC tissues and associated with depth of tumor invasion. Knockdown of IGF2BP1 inhibited ESCC cell invasion and migration as well as tumor metastasis. Importantly, INHBA was identified as a direct target of IGF2BP1 in ESCC cells, which had a role in promoting the malignant phenotypes. TCGA data and RISH analyses showed that the mRNA level of INHBA was upregaluted in ESCC tissues as well. Mechanistically, IGF2BP1 bound and stabilized INHBA mRNA and then enhanced its translation, leading to an activation of Smad2/3 signaling. Ras GTPaseactivating protein-binding protein 1 (G3BP1) was recruited by IGF2BP1 to participate in activating the signaling process, which was inhibited by the IGF2BP1 inhibitor BTYNB. Of note, IGF2BP1 mRNA expression in ESCC cells was negatively correlated with the level of its promoter methylation.

\section{Conclusions}

IGF2BP1 overexpression promotes the invasion and migration of ESCC cells by up-regulating TGF- $\beta$ Smad2/3 pathway through enhancing INHBA mRNA stability and translation, providing a potential therapeutic target for ESCC treatment.

\section{Background}

Esophageal cancer is one of the major malignancies threatening human health. Esophageal squamous cell carcinoma (ESCC) accounts for more than $90 \%$ cases of esophageal cancer in China. Most patients are diagnosed with advanced stage and the 5-year survival rate was only about $30 \%$ [1] due to invasive 
growth and distal metastasis. However, the molecular mechanisms underlying the invasion and metastasis of ESCC are still not fully understood, and so far there is no effective targeted drug for clinical treatment. Therefore, it is an urgent need to identify the key molecules affecting the invasion and metastasis of ESCC.

Insulin-like growth factor 2 mRNA binding protein 1(IGF2BP1) is a highly conserved RNA binding protein, which mainly binds mRNA and thereby affects RNA transcription, processing, translation and metabolism. IGF2BP1 overexpression often correlated to poor prognosis in a variety of cancer types including melanoma [2], breast [3], ovarian [4-6], colon [7, 8], liver [9, 10], and lung[11, 12] cancers. It has been reported that IGF2BP1 promotes cell proliferation, invasion and resistance to chemotherapeutic drugs and inhibitors by binding and stabilizing CD44, c-myc, ACTB and other oncogenic transcripts [13]. Moreover, IGF2BP1 has been proved as a N6-methyladenosine $\left(\mathrm{m}^{6} \mathrm{~A}\right)$ reader, which recognizes and binds $m^{6} A$-modified mRNA and thus enhances their stability $[14,15]$. Additionally, IGF2BP1 is termed as oncofetal protein due to its space-time specific expression pattern: predominantly expressed in embryonic development and suppressed in most of adult tissues, while re-expressed in multiple tumor types [16]. Several researches reported that, BTYNB, a selective inhibitor, can impair cell proliferation in vitro by blocking IGF2BP1 binding to its oncogenic transcripts $[17,18]$. Altogether, these studies suggest that IGF2BP1 might be a promising biomarker and therapeutic target.

We found a IGF2BP1 remarkable upregulation in ESCC tissues by IHC. But there is no information available for the role of IGF2BP1 in ESCC. In this study, we focus on the roles of IGF2BP1 overexpression on malignant phenotypes and the underlying mechanisms in ESCC cells, aiming to explore the possibility of IGF2BP1 as the biomarker and therapeutic target for the disease.

\section{Materials And Methods}

\section{Tissue specimens and cell lines}

ESCC and operative margin tissues from a total of 311 patients were procured from surgical resection specimens. All of the specimens were residual specimens collected after sampling for pathological diagnosis. All the patients received no treatment prior to surgery, and signed separate informed consent forms for sample collection and molecular analysis. The study was approved by the Ethics Committee of Cancer Institute (Hospital), CAMS \& PUMC (No. 16-084/1163).

ESCC cell lines KYSE30, KYSE70, KYSE150, KYSE180, KYSE450 and KYSE510 were generously provided by Prof. Shimada (Kyoto University, Japan); TE1, TE4 and TE10 were purchased from ATCC, and ECA109 from Cell Resource Center, Institute of Basic Medicine, Chinese Academy of Medical Sciences. All the cell lines were authenticated by Short Tandem Repeat (STR) profiling and cultured in RPMI-1640 media with $10 \%$ fetal bovine serum (FBS500-S, AusGeneX) in a humidified incubator at $37^{\circ} \mathrm{C}$ and $5 \% \mathrm{CO}_{2}$.

\section{Immunohistochemistry (IHC)}


IHC assay was conducted as reported previously [19]. Slides were incubated with primary antibody IGF2BP1(ab184305, 1:1000, Abcam) at $4^{\circ} \mathrm{C}$ overnight. The tissue microarrays were scanned with Nano Zoomer digital pathology biopsy scanner (HAMAMATSU, Japan). Immunoreactive scores were calculated as multiplying the scores of staining intensity and percentage of positive cells. The intensity was scored as follows: 0 (negative), 1 (weak), 2 (moderate), and 3 (strong); the proportion of positive cells was scored as follows: 0 (negative), 1 (1\%-20\%), 2 (21\%-50\%), 3 (51\%-100\%).

\section{RNA in situ hybridization (RISH)}

INHBA mRNA in situ hybridization was performed on $6 \mu \mathrm{m}$ thick tissue microarrays (TMA) with RNAscope 2.5 HD Reagent Kit-BROWN (322300, ACD) following the manufacturer's instruction.

\section{Plasmids and cloning}

Cloning strategies including plasmids, oligonucleotides used for PCR were summarized in Additional file 1: Table S1,2. All constructs were validated by sequencing.

\section{Transfection and lentiviral transduction}

Cells were transfected with siRNA (GenePharma, China) or overexpression plasmids using Lipofectamine 3000 Transfection Reagent (L3000015, Thermo) according to the manufacturer's instructions. Lentivirus expressing IGF2BP1-shRNA (shIGF2BP1) or no-silencing-shRNA (shNS) as negative control were used to infect ESCC cells, and the stable cell strains were selected by puromycin $(1 \mu \mathrm{g} / \mathrm{mL}$, Gibco $)$ for one week. The siRNA or shRNA sequence were provided in Additional file 1: Table S2,3.

\section{Western blotting}

Total protein was isolated using RIPA buffer (C1053, Applygen) with protease inhibitors (B14001, Bimake) and phosphatase inhibitors (B15001, bimake), and quantified with Pierce BCA Protein Assay Kit (23225, Thermo). Primary antibodies for immunoblotting included IGF2BP1 (ab184305, 1:1,000, Abcam), INHBA (ab128958, 1:1,000, Abcam), Smad2/3 (8685T, 1:1,000, CST), G3BP1(ab181150, 1:1,000, Abcam), pSmad2(s255, ab188334, 1:2,000, Abcam), METTL3 (ab195352, 1:1,000, Abcam), METTL14 (26158-1-AP, 1:500, Proteintech). GAPDH (60004-1-lg, Proteintech) was used as a loading control. Secondary antibodies were used at 1:5,000 dilution (Goat anti-mouse/rabbit IgG, HRP conjugate, SA00001-1/2, Proteintech). The signals were detected with a super enhanced chemiluminescence $(E C L)$ detection reagent (29050, Engreen).

\section{Co-immunoprecipitation-based mass spectrometry (Co-IP- MS)}

Non-denaturing lysis buffer (P0013, Beyotime) was employed to isolate the total protein lysate. $1 \mu \mathrm{g}$ of IP antibody or rabbit IgG (B900610, Proteintech) was used to immunoprecipitated $1 \mu \mathrm{g}$ of total proteins overnight at $4^{\circ} \mathrm{C}$ with rotation. Subsequently, $50 \mu \mathrm{L}$ protein $\mathrm{A} / \mathrm{G}$ magnetic beads (MedChemExpress) were introduced into the mixture and incubated in rotation for $2 \mathrm{~h}$ at $4^{\circ} \mathrm{C}$. The beads were rinsed four times with washing buffer after which they were eluted in $20 \mu \mathrm{L} 1 \times$ loading buffer and denatured at $100^{\circ} \mathrm{C}$ for 
10 min. Protein samples were then subjected to WB assay or SDA-PAGE followed by coomassie staining and sent to Shanghai Applied Protein Technology Co. Ltd. for mass spectrometry analysis..

\section{Cell viability and colony formation assay}

$1 \times 10^{3}$ cells were seeded on 96 -well plates (per well with 3 replicates in each group), and the cell viability was quantified every $24 \mathrm{~h}$ using Cell Counting Kit-8 (CK-04, Dojindo, Japan) according to the manufacturer's instruction. Absorbance was measured at $450 \mathrm{~nm}$ using by Elx 808 Microplate Reader (BioTek, USA). For colony formation assay, $1 \times 10^{3}$ cells were seeded on 6 -well plates (per well with 3 replicates in each group), fixed and stained using $0.1 \%$ crystal violet after treatment with indicated doze of BTYNB for 7-14 days.

\section{Cell apoptosis analysis}

Cells treated with $10 \mu \mathrm{M}$ BTYNB for $48 \mathrm{~h}$ were digested, collected and stained with fluorescent-labeled Annexin V and PI using Annexin V, FITC Apoptosis Detection Kit

(AD10, Dojindo). Then the apoptotic cells were identified by flow cytometry.

\section{Wound-healing assay}

Cells were seeded in six-well plates and grew until reached full confluence. $10 \mu$ tips were used to scratch a wound vertically, and the scraped cells were removed by washing with PBS. The scratches were observed and photographed at 0,12, 24, and $48 \mathrm{~h}$ after being made.

\section{Cellular migration and invasion assays}

$8 \mu \mathrm{m}$ pore 24-well Transwell plate (Corning, USA) was employed to assess the migration and invasion ability of ESCC cells. Cells were seeded into the upper compartments at a density of $1 \times 10^{5} /$ well in $200 \mu \mathrm{L}$ serum-free media, and the lower chamber were added $700 \mu \mathrm{L}$ RPIM-1640 media with $30 \%$ fetal bovine serum as a chemoattractant. After incubation 36 hours (KYSE30) or 24 hours (TE1) at $37^{\circ} \mathrm{C}$, the cells were fixed in methanol and acetone fixation solution $(1: 1, \mathrm{v} / \mathrm{v})$ and stained with $0.1 \%$ crystal violet (Sigma). The polycarbonate membranes were then cut, cells removed from apical side, and placed on slide, followed by scanning and imaging with Nano Zoomer digital pathology biopsy scanner (HAMAMATSU, Japan). The percentage of stained cell area were measured by ImageJ (Ver. 1.51j8, NIH, USA) in three random fields.

\section{Immunofluorescence (IF) staining}

Cells were fixed in $4 \%$ paraformaldehyde, permeabilized with 0.5\% Triton X-100, blocked with 5\% BSA and incubated with IGF2BP1 (sc-166344, 1:50, Santa) and G3BP1 (ab181150, 1:100, Abcam) antibody at $4{ }^{\circ} \mathrm{C}$ overnight. Then, cells were incubated with fluorescent secondary antibody (Rabbit IgG, CoraLite488 conjugate or Mouse lgG, CoraLite594 conjugate, 1:200, Proteintech) for $1 \mathrm{~h}$ at room temperature in the dark, followed by staining with DAPI (ZLI-9557, ZSGB-BIO) for $5 \mathrm{~min}$. Immunofluorescence were detected by confocal microscopy (PE Double spinning disk confocal, USA). 


\section{Xenograft assay}

Four-week-old female BALB/c nude mice (HFK Bioscience Co., LTD, Beijing, China) were purchased and randomly divided into two groups by body weight (10 per group). The mice were injected with $1 \times 10^{6}$ KYSE30 cells stably expressing shIGF2BP1 or shNon-Silencing (shNS) via the tail vein. Eight weeks later, the mice were sacrificed and the whole lung tissues were separated and fixed in Bouin's Fixative Solution (PH0976, Phygene). Then the number of lung metastases was counted, and the lung tissues were embedded in paraffin, cut into $3 \mu \mathrm{m}$ sections, and stained with hematoxylin and eosin (H\&E).

All the animal experiments were approved by the Animal Center of the Institute of National Cancer Center/Cancer Hospital, CAMS \& PUMC (NCC2019A016).

\section{Quantitative and Semi-quantitative RT-PCR}

Total RNA was isolated using RNApure Tissue \& Cell Kit (CW0506, Cwbiotech) following the manufacturer's instructions, and cDNA was synthesized using HiFiScript cDNA Synthesis Kit (CW2569M, Cwbiotech). Then quantitative real-time PCR was performed using TB Green ${ }^{\text {TM }}$ Premix Ex Taq kit (RR420A, TaKaRa) on an ABI QuantStudio DX real-time PCR System (ABI, USA). GAPDH was used as loading control, and the relative expression levels of mRNA were assessed through the comparative threshold cycle method $\left(2^{-\Delta \Delta C t}\right)$. All primers used in this study are listed in Additional file 1: Table S4.

\section{RNA co-Immunoprecipitation combined with high- throughput sequencing (RIP-seq)}

RIP was performed using EZ-Magna RIP Kit (17-701, Millipore). Briefly, $2 \times 10^{7}$ cells were harvested and re-suspended in complete RIP Lysis Buffer (RIP lysis buffer supplemented with protease inhibitor cocktail and RNase inhibitor) on ice. one tenth of the supernatant of RIP lysate was saved as input. $5 \mu \mathrm{g}$ of IGF2BP1 antibody (RN007P, MBL) or negative control normal rabbit IgG (PP64B, Millipore) was conjugated to protein $A / G$ magnetic beads (MedChemExpress), followed by incubation with the RIP lysate in RIP Immunoprecipitation buffer (RIP wash buffer supplemented with 0.5 M EDTA and RNase inhibitor) for $4 \mathrm{~h}$ at $4^{\circ} \mathrm{C}$. Then the immunoprecipitate was digested by proteinase $\mathrm{K}$ buffer. RNA was finally purified with TRIzol reagent (Invitrogen) and analyzed by qPCR or RNA-seq (Wuhan Seqhealth Tech Co. Ltd.). The sequences of primers for qPCR are described in Additional file 1: Table S4.

\section{Biotin RNA pull-down assay}

Cell lysates were prepared in pulldown buffer $(150 \mathrm{mM} \mathrm{KCL}, 0.5 \% \mathrm{NP}-40,25 \mathrm{mM}$ Tris- $\mathrm{HCl}$ pH7.4, $0.5 \mathrm{mM}$ DTT, supplemented with proteasome inhibitors and $80 \mathrm{U} / \mathrm{mL}$ RNase inhibitors) and incubated with biotinlabelled DNA probes at $4^{\circ} \mathrm{C}$ for $2 \mathrm{~h}$. Then the RNA-protein complex was immunoprecipitated with streptavidin magnetic beads (MedChemExpress) after an additional $2 \mathrm{~h}$ incubation at $4^{\circ} \mathrm{C}$. The complex was divided into two equal portions. One was added to TRIzol reagent for RNA isolation followed by RTPCR; the other was eluted with $1 \times$ protein loading buffer for Western blotting. 1/50 of the cell lysates were 
saved as input for RT-PCR and WB analysis. The sequences of biotin-labelled DNA probes against INHBA were provided in Additional file 1: Table S5.

\section{RNA stability assay}

ESCC cells stably expressing shIGF2BP1 or shNS were seeded into 12-well plates to get $80 \%$ confluency after $24 \mathrm{~h}$. Then cells were treated with actinomycin $D$ (ActD, $5 \mu \mathrm{g} / \mathrm{mL}$ ) for $0,2,4 \mathrm{~h}$, respectively. Total RNA was then extracted and analyzed by quantitative real-time PCR, and the relative level of INHBA mRNA at each time point was quantified, and GAPDH was used as loading control. The mRNA half-life was estimated according to the previous description[20].

\section{Gene-specific $\mathrm{m}^{6} \mathrm{~A}$ qPCR}

$100 \mu \mathrm{g}$ of total RNA was extracted and one tenth of the RNA was saved as the input control. Protein A/G Magnetic Beads (MCE) were prewashed and incubated with $5 \mu \mathrm{g}$ of anti-m ${ }^{6} \mathrm{~A}$ antibody (ABE572, Millipore) or rabbit IgG (PP64B, Millipore) for $2 \mathrm{~h}$ at $4^{\circ} \mathrm{C}$ with rotation. Then the antibody-conjugated beads were mixed with total RNA in $1 \times$ binding buffer supplemented with RNase inhibitors for additional $2 \mathrm{~h}$ at $4^{\circ} \mathrm{C}$ with rotation. The methylated mRNAs were eluted with elution buffer (10 mL $0.1 \mathrm{M} \mathrm{DTT}, 0.44 \mathrm{~g} \mathrm{NaCl}$, $2.5 \mathrm{~mL}$ pH7.5 $1 \mathrm{M}$ Tris-HCl, 0.1 mL 0.5 M EDTA, 0.5 mL 10\% SDS, $10 \mu \mathrm{L}$ Inhibitor, ddH $\mathrm{d}_{2} \mathrm{O}$ up to $50 \mathrm{~mL}$ ) and recovered with RNeasy Micro Kit (74004, Qiagen), further analyzed by RT-PCR along with input control.

\section{Methylation specific PCR (MSP-PCR)}

Genomic DNA of ESCC cells was extracted using the QIAamp DNA Mini Kit (QIAGEN) and transformed with Epitect Fast DNA Bisulfite Kit (QIAGEN). PCR amplification was performed with methylated primers (M primer) and unmethylated primers (U primer) by using the transformed DNA as a template. The sequences of $\mathrm{M}$ and $\mathrm{U}$ primer pairs against the first intron were provided in Additional file 1: Table S6.

\section{Databases analysis}

The Cancer Genome Atlas (TCGA) (https://cancergenome.nih.gov) datasets were employed to detect the mRNA expression of IGF2BP1 and INHBA in ESCC and non-malignant tissues; the Wanderer interactive viewer (http://maplab.imppc.org/wanderer/) was used to detect the IGF2BP1 and INHBA mRNA expression in head and neck squamous cell carcinoma (HNSCC), lung squamous cell carcinoma (LUSC) and cervical squamous cell carcinoma (CESC), compared with the normal tissues. The expression for IGF2BP1 mRNA in all major tissues and organs in the human body was analyzed in HPA database (https://www.proteinatlas.org/).

\section{Statistical analysis}

IBM SPSS Statistics 23.0 software was applied for data analysis, and $P<0.05$ was considered as statistically significant. Fisher's exact test was used to assess the IHC scores difference between ESCC tissues and adjacent non-cancerous specimens. The correlation between protein expression level and clinicopathological parameters was analyzed by Pearson Chi-square test. Comparison between two 
groups was performed by independent sample T test, and one-way ANOVA test was used for multiple comparisons. RStudio software (1.1463) was used for GO and Pathway enrichment analysis.

Additional Materials and Methods are described in Additional files.

\section{Results}

\section{IGF2BP1 is highly expressed in ESCC tissues and is associated with depth of tumor invasion}

We examined the protein expression level of IGF2BP1 in 311 cases of ESCC tissues and 9 cases of adjacent normal tissues on TMA by IHC technique. The results showed that IGF2BP1 was highly expressed in ESCC tissues (155/311,49.8\%), but was barely be detectable in normal esophageal epithelia (Fig. 1A, Table 1). And the positive staining was predominant in the cytoplasm of ESCC cells. A higher IGF2BP1 expression level was positively correlated with the depth of tumor invasion $\left(T_{1-2}\right.$ versus $\left.T_{3-4}\right)$, but no significant differences were found in other clinicopathological features, such as sex, age, histologic grade, lymph node metastasis and clinical stage (Table 1). 
Table 1

The association between IGF2BP1 expression and the clinicopathological variables

\begin{tabular}{|c|c|c|c|c|}
\hline \multirow[t]{2}{*}{ Clinicopathologic } & \multirow[t]{2}{*}{ Case $(\mathrm{N})$} & \multicolumn{3}{|c|}{ IGF2BP1 expression } \\
\hline & & Positive N (\%) & $\chi^{2}$ & P-value \\
\hline Cases & 311 & $155(49.84)$ & & 0.004 \\
\hline \multicolumn{5}{|l|}{ age } \\
\hline$<60$ & 98 & $44(44.90)$ & 0.361 & \multirow[t]{2}{*}{0.548} \\
\hline$\geq 60$ & 213 & $111(52.10)$ & & \\
\hline \multicolumn{5}{|l|}{ gender } \\
\hline male & 225 & $114(50.70)$ & 0.223 & \multirow[t]{2}{*}{0.637} \\
\hline female & 86 & $41(47.70)$ & & \\
\hline \multicolumn{5}{|l|}{ Grade } \\
\hline G3 & 67 & $35(52.24)$ & 3.724 & \multirow[t]{3}{*}{0.155} \\
\hline G2 & 200 & $104(52.00)$ & & \\
\hline G1 & 44 & $16(36.36)$ & & \\
\hline \multicolumn{5}{|l|}{ pT } \\
\hline T1-2 & 84 & $33(39.29)$ & 5.127 & \multirow[t]{2}{*}{0.024} \\
\hline T3-4 & 227 & $122(53.74)$ & & \\
\hline \multicolumn{5}{|l|}{$\mathrm{pN}$} \\
\hline NO & 161 & $81(50.31)$ & 0.03 & \multirow[t]{2}{*}{0.863} \\
\hline N1-4 & 150 & $74(49.33)$ & & \\
\hline \multicolumn{5}{|l|}{ Stage } \\
\hline प & 30 & $14(46.67)$ & 0.493 & \multirow[t]{4}{*}{0.92} \\
\hline प & 143 & $71(49.65)$ & & \\
\hline प & 120 & $62(51.67)$ & & \\
\hline$\square$ & 18 & $8(44.44)$ & & \\
\hline
\end{tabular}

Furthermore, RNA-seq data obtained from the TCGA database revealed that the mRNA expression level of IGF2BP1 was elevated in ESCC specimens compared with that in normal tissues (Fig. 1B), which was consistent with the IHC results. 


\section{IGF2BP1 promotes ESCC cells migration, invasion and metastasis}

To identify the role of IGF2BP1 in ESCC, the expression of IGF2BP1 in 11 ESCC cell lines was detected by Western blot. We found that IGF2BP1 had a higher expression in KYSE30, KYSE450, KYSE140 and TE1, but was almost undetectable in KYSE70, KYSE150 and TE10 cells (Fig. 2A). KYSE30 and TE1 were applied to the functional study as cell models. After transient knockdown of IGF2BP1 mediated by small interfering RNA (siRNA), there was no significant change in cell growth with seven days compared with the control group (Fig. 2B). However, the migration and invasion ability of both the two cell lines were substantially repressed after transfection in the transwell (Fig. 2C) and wound healing assays (Fig. 2D).

Next, KYSE30 cells stably expressing shRNA of IGF2BP1 (shIGF2BP1) or non-silencing shRNA (shNS) conducted by lentivirus infection were injected to nude mice via the tail veins. The formation of lung metastatic tumors was observed in both groups six weeks later, but the number of lung metastases in the shIGF2BP1 group was significantly reduced, and the metastatic nodules were noticeably small (Fig. 2E).

\section{IGF2BP1 increases INHBA mRNA stability, promoting ESCC cells migration and invasion}

Given that IGF2BP1 is an RNA binding protein, we performed RIP-seq in KYSE30 cells to identify its potential RNA targets. The results showed that most of the IGF2BP1-binding sites were highly enriched in coding regions (CDS) compared to the input control, indicating that IGF2BP1 mainly regulates gene expression by binding CDS (Additional file 2: Figure S1). We selected and verified several genes related to cell migration and invasion through GO analysis. Notably, the mRNA abundance of INHBA was remarkably reduced after IGF2BP1 knockdown in KYSE30, KYSE450 and TE1 cells (Fig. 3A). RIP-PCR and RNA-pulldown using biotin-labeled DNA probe against INHBA mRNA further confirmed the interaction between IGF2BP1 protein and INHBA transcript (Fig. 3B, C). Furthermore, silencing IGF2BP1 downregulated the protein expression of INHBA and Smad2/3 in KYSE30 and TE1 cells (Fig. 3D).

Previous studies have shown that IGF2BP1 can recognize N6-methyladenosine $\left(\mathrm{m}^{6} \mathrm{~A}\right)$ and enhance mRNA stability and translation in an $\mathrm{m}^{6} \mathrm{~A}$-dependent manner $[14,15]$. Therefore, it is highly possible that IGF2BP1 stabilized the mRNA of INHBA after their binding. RNA stability assay showed that the half-life of INHBA mRNA was significantly shortened after knockdown of IGF2BP1 in KYSE30 and TE1 cells (Fig. $3 E$ ). As the $m^{6} A$ writers-METTL3 or METTL14 was repressed by siRNAs, the INHBA protein levels were decreased in both cell lines without affecting IGF2BP1 (Fig. 3F). Gene-specific $\mathrm{m}^{6} \mathrm{~A}$ qPCR further confirmed that INHBA was regulated by $\mathrm{m}^{6} \mathrm{~A}$ modification (Fig. $3 \mathrm{G}$ ).

INHBA is a member of the transforming growth factor $\beta$ (TGF- $\beta$ ) superfamily, which is closely associated with tumor invasion and metastasis. We employed transwell assay to assess the effects of INHBA on invasive and migratory phenotypes in ESCC cells. We observed that cell invasion and migration were inhibited after knockdown of INHBA by siRNA (Fig. 3H), while transient overexpression of INHBA in cells 
with stably knockdown of IGF2BP1 partially restored the inhibition (Fig. 3I). Western blotting showed that Smad2/3 expression was repressed by IGF2BP1 depletion in KYSE30 and TE1 cells, while the IGF2BP1 knockdown-induced Smad2/3 decrease was reversed by INHBA overexpression (Fig. 3J).

\section{IGF2BP1 activates INHBA-Smad2/3 signaling by recruiting G3BP1}

It has been reported that IGF2BPs interacts with other RNA-binding proteins (RBPs) to regulate mRNA targets [21, 22]. For further elucidating the functional mechanism of IGF2BP1 in ESCC cells, we adopted Co-IP-MS to investigate interactive partners of IGF2BP1 (Additional file 3: Figure S2). A total of 227 potential proteins were identified, $47 \%$ of them participate in RNA regulation. GO and Pathway enrichment analysis revealed that most of them involved in RNA processing, localization, stability, metabolism, and transport and RNA splicing (Fig. 4A-B).

We selected G3BP1 as a candidate owing to its oncogenic role in other tumors and the involvement in RNA binding. Endogenous G3BP1 was immunoprecipitated by IGF2BP1 antibody using Co-IP assay, and vice versa (Fig. 4C). Immunofluorescence by confocal microscopy confirmed the cytoplasmic colocalization of G3BP1 and IGF2BP1 in KYSE30 and TE1 cells (Fig. 4D). Moreover, Western blotting showed that silencing G3BP1 by siRNA led to a decrease of INHBA and Smad2/3, and a slight reduction in IGF2BP1 (Fig. 4E).

\section{INHBA is significantly upregulated in ESCC, HNSC and invasive breast cancer}

We then analyzed the TCGA transcriptome sequencing datasets, and observed that the mRNA of IHBA was also increased in ESCC tissues (Fig. 5A), which was consistent with its functional role in ESCC and posttranscriptional regulation by IGF2BP1. RISH on tissue microarrays further confirmed the RNA-Seq results. More importantly, INHBA was mainly distributed in the peripheral tumor cells of cancer nests and the stroma of ESCC tissues but negative in the normal esophageal epithelia and stroma (Fig. 5B). Besides, in HNSC and invasive breast cancer, INHBA mRNA was significantly elevated as well based on TCGA datasets (http://maplab.imppc.org/wanderer/) (Fig. 5C).

\section{Small molecule inhibitor BTYNB significantly inhibits the invasion, migration and proliferation of ESCC cells in vitro}

Currently, there are no inhibitors in clinical trials that directly target IGF2BP1. Previous studies reported a small molecule drug, BTYNB, could inhibit the binding of IGF2BP1 to c-Myc mRNA $[17,23]$. BTYNB also impairs cell proliferation in vitro by blocking $\beta$-TRCP1, E2F and other transcripts $[17,18]$. So, it is reasonable to speculate that BTYNB might interfere with the IGF2BP1-driven malignant phenotypes in ESCC cells. We tested BTYNB with transwell assay by adding the drug into the lower chamber, and found that the migration and invasion of KYSE30 and TE1 cells were significantly inhibited in $36 \mathrm{~h}$ or $24 \mathrm{~h}$, respectively (Fig. 6A). Meanwhile, cell viability and colony formation were impaired in a dose-dependent 
manner (Fig. 6B-C). Besides, a $48 \mathrm{~h}$ exposure to BTYNB increased the number of apoptotic cells (Fig. 6D), and INHBA and Smad2/3 expression were decreased after BTYNB treatment (Fig. 6E).

\section{Elevated expression of IGF2BP1 in ESCC cells is associated with hypomethylation of its first intron}

We further detected possible dysregulation of IGF2BP1 in other types of SCCs, and also found a higher IGF2BP1 mRNA in HNSCC, LUSC, and CESC compared with that in the corresponding normal tissues (http://maplab.imppc.org/wanderer/, Fig. 7A). Moreover, we observed a high degree of consistency between the mRNA and protein levels of IGF2BP1 in 10 ESCC cell lines (Fig. 7B). And interestingly, according to the HPA (Human Protein Atlas) database, IGF2BP1 was almost absent in normal esophageal tissues and other normal tissues except in the embryo and reproductive system (Fig. 7C). To uncover the mechanism of IGF2BP1 mRNA upregulation in ESCC cells, we examined the methylation status of this gene. Three pairs of primers (methylated and unmethylated primers) were designed for detecting distinct CG sites in the first intron of IGF2BP1, and MSP-PCR was conducted in both high and Iow IGF2BP1 expression cell lines, respectively. The results showed that IGF2BP1 gene was hypomethylated in cell lines with high IGF2BP1 expression, but hypermethylated in low IGF2BP1 cell lines except for TE10 (Fig. 7D).

\section{Discussion}

The insulin-like growth factor-2 mRNA-binding protein family (IGF2BPs), composed of IGF2BP1, IGF2BP2 and IGF2BP3, has a crucial role in early embryonic development. IGF2BP1 and IGF2BP3 are demonstrated as oncofetal proteins, because they are silent in adult organs, except in reproductive system $[13,16,24]$. In contrast, IGF2BP2 is the only expressive IGF2BP in most adult tissues. It has been observed that IGF2BP1 and IGF2BP3 are re-expressed in many types of tumors, and IGF2BP2 was also found to be excessive in malignances due to genomic amplification according to pan-cancer analysis with TCGA data. Growing evidences support the pro-oncogenic roles of these RNA-binding proteins in cancer progression by influencing their RNA target fate [25].

However, few studies on IGF2BP1 have been reported in ESCC. Herein we discovered a remarkable high expression of IGF2BP1 at both the mRNA and protein levels, indicating a transcriptional dysregulation in ESCC. More importantly, our functional and mechanism investigations revealed that IGF2BP1 facilitated the migration, invasion and metastasis of ESCC cells via activating INHBA-Smad2/3 cascade. INHBA, a member of TGF- $\beta$ superfamily, has been reported to be overexpressed in multiple types of cancers including ESCC and associated with poor prognosis [26-31]. Consistently, our analyses to the TCGA data and RISH on TMAs demonstrated the upregulation of INHBA at the transcriptional level in ESCC tissues. Besides, we found a high upregulation of INHBA mRNA in HNSC and invasive breast cancer as well. Combined with the spatial distribution of INHBA mRNA in ESCC tissues, we speculate that INHBA may play an important role in cell invasion and migration. Although the roles of INHBA in cancer is controversial, the majority favors its oncogenic effects. Seder et al. reported that INHBA promoted cell 
proliferation and was regulated by promoter demethylation [32] in ESCC cells. In the present study, we identified INHBA as a direct target of IGF2BP1, and had a function in tumor invasion and metastasis induced by IGF2BP1. Mechanically, IGF2BP1 bound and stabilized INHBA mRNA, thus enhancing the translation of INHBA. Moreover, as an $\mathrm{m}^{6} \mathrm{~A}$ reader proved by a recent research, IGF2BP1 preferentially recognizes $m^{6} \mathrm{~A}$-modified $m R N A s$ and promotes their stability in a $m^{6} \mathrm{~A}$ dependent manner [33]. We indeed observed that INHBA mRNA was $\mathrm{m}^{6} \mathrm{~A}$ modified, and the turnover of INHBA was $\mathrm{m}^{6} \mathrm{~A}$ dependent. So it is probably that mRNA methylation is required in the regulation of INHBA by IGF2BP.

Like other RNA-binding proteins (RBPs), IGF2BP1 participates in forming ribonucleoprotein (RNP) granules that regulate mRNA translation, localization, and turnover [21]. We identified 107 proteins related to RNA processing by mass spectrometry, accounting for $47 \%$ of all interactive proteins of IGF2BP1 in ESCC cells. We found that G3BP1 was a partner of IGF2BP1 and contributed to regulate INHBA-Smad2/3 signaling positively. G3BP1 contains two C-terminal motifs (associated with RNA binding) and an RNA recognition motif (RRM). It has been demonstrated that G3BP1 promotes stress-induced RNA granule interactions to preserve polyadenylated mRNA [34]. Meanwhile, G3BP1 involved in protein degradation by stably associated with USP10 deubiquitinase [35]. Therefore, G3BP1 might activate the IGF2BP1-INHBASmad2/3 signaling either by promoting protein stability or by enhancing RNA stability. The specific detail on how G3BP1 facilitates IGF2BP1 to elevate INHBA still need further investigation.

In view of a oncofetal pattern of expression, IGF2BP1 could be a promising target, making it possible to specifically target tumor cells without disturbing the noncancerous tissues. Several studies have demonstrated that BTYNB, a structure-specific inhibitor, could block the binding of IGF2BP1 to its oncogenic target mRNA, thus disrupt their interaction $[17,18]$. We evaluated the efficacy of BTYNB in vivo, and found that the typical malignant phenotypes of high IGF2BP1 expression ESCC cells were sharply repressed, and apoptotic cells were increased after BTYNB exposure. At the molecular level, BTYNB treatment led to an attenuated IGF2BP1-INHBA-Smad2/3 signaling. These data imply that IGF2BP1 is a potential molecular target for the therapy of ESCC.

Little information is available about how the expression of IGF2BP1 is modulated at the transcriptional and post-transcriptional level. It has been proposed that IGF2BP1 transcription was induced by $\beta$-catenin [36] and enhanced by c-Myc through a positive feed-back loop [37]. Besides, let-7 could regulate IGF2BP1 post-transcriptionally [38]. Our observation linked genomic hypomethylation in the first intron to high IGF2BP1 expression, providing a new perspective on aberrant transcription of this gene.

\section{Conclusions}

The present study reveals for the first time that IGF2BP1 plays a pivotal role on the invasion and migration of ESCC cells via activating IHNBA-Smad2/3 signaling pathway. Our data indicate that IGF2BP1 may serve as a promising biomarker and an attractive target for the disease.

\section{Abbreviations}


ESCC: Esophageal squamous cell carcinoma; SCC: Squamous cell carcinoma; HNSCC :Head and neck squamous cell carcinoma; LUSC: Lung squamous cell carcinoma; CESC: Cervical squamous cell carcinoma; IHC: Immunohistochemistry; RISH: RNA in situ hybridization; TMA: Tissue microarray; H\&E staining: Haematoxylin \& eosin staining; IF: Immunofluorescence; siRNA: small interfering RNA; RIP: RNA co-Immunoprecipitation; RT-PCR: Reverse transcription-polymerase chain reaction; WB: Western blotting; Co-IP-MS: Co-immunoprecipitation-mass spectrometry; MSP-PCR: Methylation specific PCR; TCGA: The Cancer Genome Atlas; HPA: Human Protein Atlas; GO: Gene Ontology; KEGG: Kyoto Encyclopedia of Genes and Genomes; RBPs: RNA-binding proteins; IGF2BPs: Insulin-like growth factor-2 mRNA-binding protein family; CDS: Coding sequence; $\mathrm{m}^{6} \mathrm{~A}$ : N6-methyladenosine; TGF- $\beta$ : Transforming growth factor $\beta$; G3BP1: Ras GTPase-activating protein-binding protein 1; RNP: Ribonucleoprotein; RRM: RNA recognition motif; ActD: actinomycin D.

\section{Declarations}

\section{Funding}

This study was supported by National Key Research and Development Program of China (2017YFE0112100), National Natural Science Foundation of China (81930077), Chinese Academy of Medical Sciences (CAMS) Fundamental Research Funds for the Central Public-interest Scientific Institution (2018RC310008), and Potential Development Projects of Cancer Hospital, CAMS (PY2018B01).

\section{Availability of data and materials}

The datasets (TCGA.ESCA.sampleMap/HiSeqV2) analyzed during the current study are available in the UCSC Xena TCGA hub repository, https://tcga.xenahubs.net.

\section{Authors' contributions}

JJH and MRW conceived and supervised the study. JJW contributed to the study design and performed most of the assays. DXC and JJW performed animal experiments. YC and JJW conducted immunohistochemistry assay. XX and YC provided necessary materials. JJW interpreted the data and drafted the manuscript. MRW revised the manuscript. All authors read and approved the final manuscript.

\section{Ethics approval}

The study was approved by the Ethics Committee of National Cancer Center/Cancer Institute (Hospital), CAMS \& PUMC (No. 16-084/1163). All the animal experiments were approved by the Animal Center of the Institute of National Cancer Center/Cancer Hospital, CAMS \& PUMC (NCC20195A0136).

\section{Consent for publication}

Not applicable. 


\section{Competing interests}

The authors declare that they have no competing interests.

\section{References}

1. Zeng H, Chen W, Zheng R, Zhang S, Ji JS, Zou X, et al. Changing cancer survival in China during 2003-15: a pooled analysis of 17 population-based cancer registries. Lancet Glob Health. 2018;6(5):e555e67.

2. Elcheva I, Tarapore RS, Bhatia N, Spiegelman VS. Overexpression of mRNA-binding protein CRD-BP in malignant melanomas. Oncogene. 2008;27(37):5069-74.

3. Gu W, Katz Z, Wu B, Park HY, Li D, Lin S, et al. Regulation of local expression of cell adhesion and motility-related mRNAs in breast cancer cells by IMP1/ZBP1. J Cell Sci. 2012;125(Pt 1):81-91.

4. Bley N, Schott A, Muller S, Misiak D, Lederer M, Fuchs T, et al. IGF2BP1 is a targetable SRC/MAPKdependent driver of invasive growth in ovarian cancer. RNA Biol. 2021;18(3):391-403.

5. Kobel M, Weidensdorfer D, Reinke C, Lederer M, Schmitt WD, Zeng K, et al. Expression of the RNAbinding protein IMP1 correlates with poor prognosis in ovarian carcinoma. Oncogene. 2007;26(54):75849.

6. Fan L, Lin Y, Lei H, Shu G, He L, Yan Z, et al. A newly defined risk signature, consisting of three $\mathrm{m}(6)$ A RNA methylation regulators, predicts the prognosis of ovarian cancer. Aging (Albany NY). 2020;12(18):18453-75.

7. Zhang XL, Li KJ, Feng JX, Liu GJ, Feng YL. Blocking the IGF2BP1-promoted glucose metabolism of colon cancer cells via direct de-stabilizing mRNA of the LDHA enhances anticancer effects. Mol Ther Nucleic Acids. 2021;23:835-46.

8. Dimitriadis E, Trangas T, Milatos S, Foukas PG, Gioulbasanis I, Courtis N, et al. Expression of oncofetal RNA-binding protein CRD-BP/IMP1 predicts clinical outcome in colon cancer. Int J Cancer. 2007;121(3):486-94.

9. Zhang J, Hu K, Yang YQ, Wang Y, Zheng YF, Jin Y, et al. LIN28B-AS1-IGF2BP1 binding promotes hepatocellular carcinoma cell progression. Cell Death Dis. 2020;11(9):741.

10. Hammerle M, Gutschner T, Uckelmann H, Ozgur S, Fiskin E, Gross M, et al. Posttranscriptional destabilization of the liver-specific long noncoding RNA HULC by the IGF2 mRNA-binding protein 1 (IGF2BP1). Hepatology. 2013;58(5):1703-12.

11. Kato T, Hayama S, Yamabuki T, Ishikawa N, Miyamoto M, Ito T, et al. Increased expression of insulin-like growth factor-II messenger RNA-binding protein 1 is associated with tumor progression in 
patients with lung cancer. Clin Cancer Res. 2007;13(2 Pt 1):434-42.

12. Zhang J, Luo W, Chi X, Zhang L, Ren Q, Wang H, et al. IGF2BP1 silencing inhibits proliferation and induces apoptosis of high glucose-induced non-small cell lung cancer cells by regulating Netrin-1. Arch Biochem Biophys. 2020;693:108581.

13. Huang X, Zhang H, Guo X, Zhu Z, Cai H, Kong X. Insulin-like growth factor 2 mRNA-binding protein 1 (IGF2BP1) in cancer. J Hematol Oncol. 2018;11(1):88.

14. Wang X, Lu Z, Gomez A, Hon GC, Yue Y, Han D, et al. N6-methyladenosine-dependent regulation of messenger RNA stability. Nature. 2014;505(7481):117-20.

15. Huang H, Weng H, Sun W, Qin X, Shi H, Wu H, et al. Recognition of RNA N(6)-methyladenosine by IGF2BP proteins enhances mRNA stability and translation. Nat Cell Biol. 2018;20(3):285-95.

16. Bell JL, Wachter K, Muhleck B, Pazaitis N, Kohn M, Lederer M, et al. Insulin-like growth factor 2 mRNA-binding proteins (IGF2BPs): post-transcriptional drivers of cancer progression? Cell Mol Life Sci. 2013;70(15):2657-75.

17. Mahapatra L, Andruska N, Mao C, Le J, Shapiro DJ. A Novel IMP1 Inhibitor, BTYNB, Targets c-Myc and Inhibits Melanoma and Ovarian Cancer Cell Proliferation. Transl Oncol. 2017;10(5):818-27.

18. Muller S, Bley N, Busch B, Glass M, Lederer M, Misiak C, et al. The oncofetal RNA-binding protein IGF2BP1 is a druggable, post-transcriptional super-enhancer of E2F-driven gene expression in cancer. Nucleic Acids Res. 2020;48(15):8576-90.

19. Shi F, Shang L, Pan BQ, Wang XM, Jiang YY, Hao JJ, et al. Calreticulin promotes migration and invasion of esophageal cancer cells by upregulating neuropilin-1 expression via STAT5A. Clin Cancer Res. 2014;20(23):6153-62.

20. Chen CY, Ezzeddine N, Shyu AB. Messenger RNA half-life measurements in mammalian cells. Methods Enzymol. 2008;448:335-57.

21. Jonson L, Vikesaa J, Krogh A, Nielsen LK, Hansen T, Borup R, et al. Molecular composition of IMP1 ribonucleoprotein granules. Mol Cell Proteomics. 2007;6(5):798-811.

22. Jonson L, Christiansen J, Hansen TVO, Vikesa J, Yamamoto Y, Nielsen FC. IMP3 RNP safe houses prevent miRNA-directed HMGA2 mRNA decay in cancer and development. Cell Rep. 2014;7(2):539-51.

23. Mahapatra L, Mao C, Andruska N, Zhang C, Shapiro DJ. High-throughput fluorescence anisotropy screen for inhibitors of the oncogenic mRNA binding protein, IMP-1. J Biomol Screen. 2014;19(3):427-36.

24. Lederer M, Bley N, Schleifer C, Huttelmaier S. The role of the oncofetal IGF2 mRNA-binding protein 3 (IGF2BP3) in cancer. Semin Cancer Biol. 2014;29:3-12. 
25. Degrauwe N, Suva ML, Janiszewska M, Riggi N, Stamenkovic I. IMPs: an RNA-binding protein family that provides a link between stem cell maintenance in normal development and cancer. Genes Dev. 2016;30(22):2459-74.

26. Lyu S, Jiang C, Xu R, Huang Y, Yan S. INHBA upregulation correlates with poorer prognosis in patients with esophageal squamous cell carcinoma. Cancer Manag Res. 2018;10:1585-96.

27. Bashir M, Damineni S, Mukherjee G, Kondaiah P. Activin-A signaling promotes epithelialmesenchymal transition, invasion, and metastatic growth of breast cancer. NPJ Breast Cancer. 2015;1:15007.

28. Okano M, Yamamoto H, Ohkuma H, Kano Y, Kim H, Nishikawa S, et al. Significance of INHBA expression in human colorectal cancer. Oncol Rep. 2013;30(6):2903-8.

29. Wang Q, Wen YG, Li DP, Xia J, Zhou CZ, Yan DW, et al. Upregulated INHBA expression is associated with poor survival in gastric cancer. Med Oncol. 2012;29(1):77-83.

30. Seder CW, Hartojo W, Lin L, Silvers AL, Wang Z, Thomas DG, et al. Upregulated INHBA expression may promote cell proliferation and is associated with poor survival in lung adenocarcinoma. Neoplasia. 2009;11(4):388-96.

31. Lee HY, Li CC, Huang CN, Li WM, Yeh HC, Ke HL, et al. INHBA overexpression indicates poor prognosis in urothelial carcinoma of urinary bladder and upper tract. J Surg Oncol. 2015;111(4):414-22.

32. Seder CW, Hartojo W, Lin L, Silvers AL, Wang Z, Thomas DG, et al. INHBA overexpression promotes cell proliferation and may be epigenetically regulated in esophageal adenocarcinoma. $\mathrm{J}$ Thorac Oncol. 2009;4(4):455-62.

33. Huang H, Weng H, Sun W, Qin X, Shi H, Wu H, et al. Author Correction: Recognition of RNA N(6)methyladenosine by IGF2BP proteins enhances mRNA stability and translation. Nat Cell Biol. 2018;20(9):1098.

34. Aulas A, Caron G, Gkogkas CG, Mohamed NV, Destroismaisons L, Sonenberg N, et al. G3BP1 promotes stress-induced RNA granule interactions to preserve polyadenylated mRNA. J Cell Biol. 2015;209(1):73-84.

35. Meyer C, Garzia A, Morozov P, Molina H, Tuschl T. The G3BP1-Family-USP10 Deubiquitinase Complex Rescues Ubiquitinated 40S Subunits of Ribosomes Stalled in Translation from Lysosomal Degradation. Mol Cell. 2020;77(6):1193-205 e5.

36. Noubissi FK, Elcheva I, Bhatia N, Shakoori A, Ougolkov A, Liu J, et al. CRD-BP mediates stabilization of betaTrCP1 and c-myc mRNA in response to beta-catenin signalling. Nature. 2006;441(7095):898-901. 
37. Noubissi FK, Nikiforov MA, Colburn N, Spiegelman VS. Transcriptional Regulation of CRD-BP by Cmyc: Implications for c-myc Functions. Genes Cancer. 2010;1(10):1074-82.

38. Boyerinas B, Park SM, Murmann AE, Gwin K, Montag AG, Zillhardt M, et al. Let-7 modulates acquired resistance of ovarian cancer to Taxanes via IMP-1-mediated stabilization of multidrug resistance 1. Int J Cancer. 2012;130(8):1787-97.

\section{Figures}


A

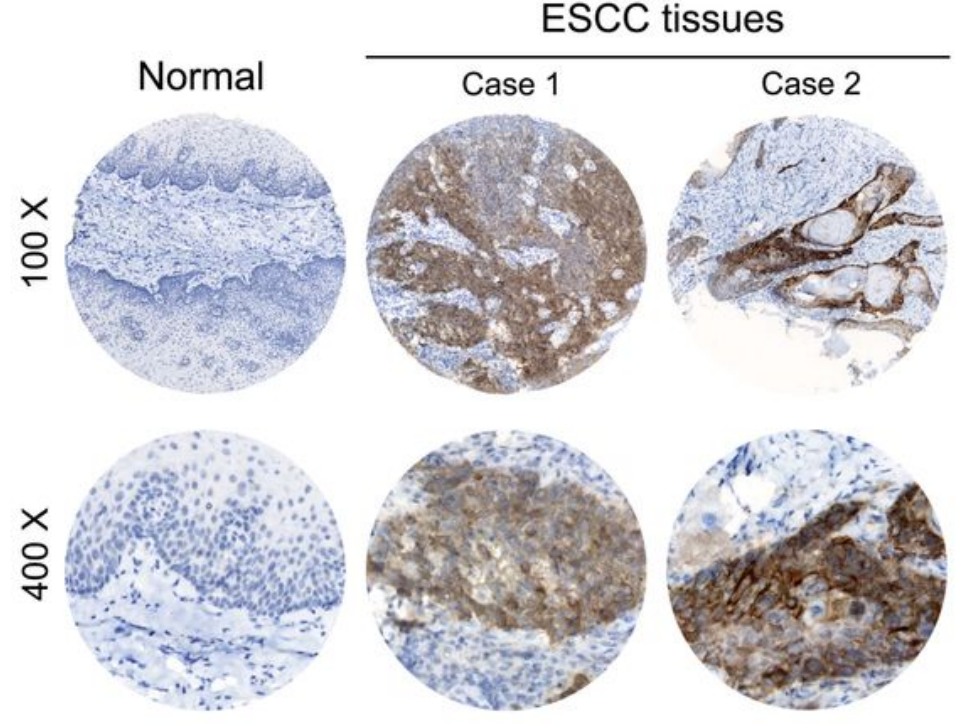

B

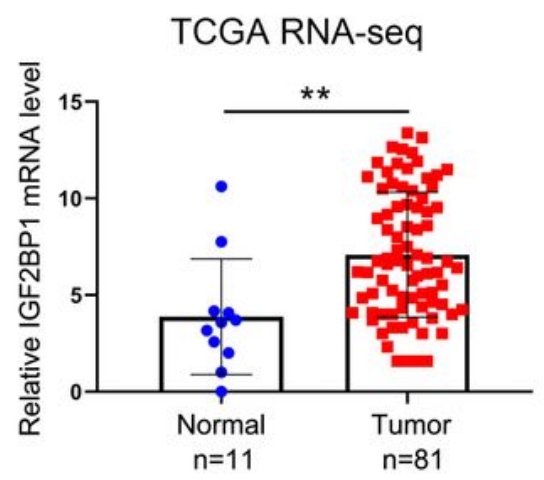

\section{Figure 1}

IGF2BP1 is overexpressed in ESCC tissues. A. Representative IHC staining of IGF2BP1 in ESCC and adjacent non-malignant tissues. B. IGF2BP1 mRNA level in ESCC patients and normal tissues in TCGA database. ${ }^{*} \mathrm{P}<0.01$. 
A



C

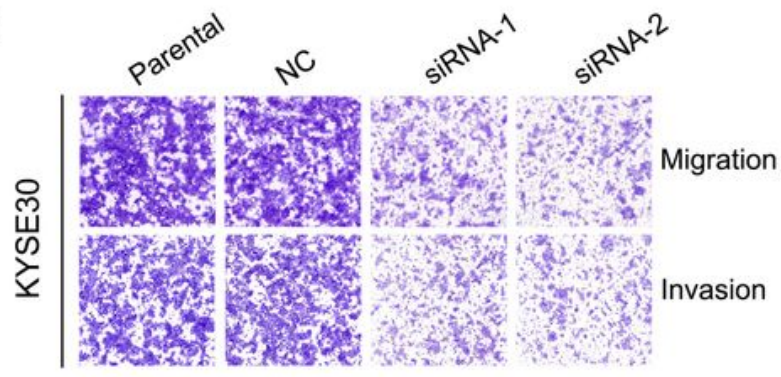

$\overline{\underline{H}}$
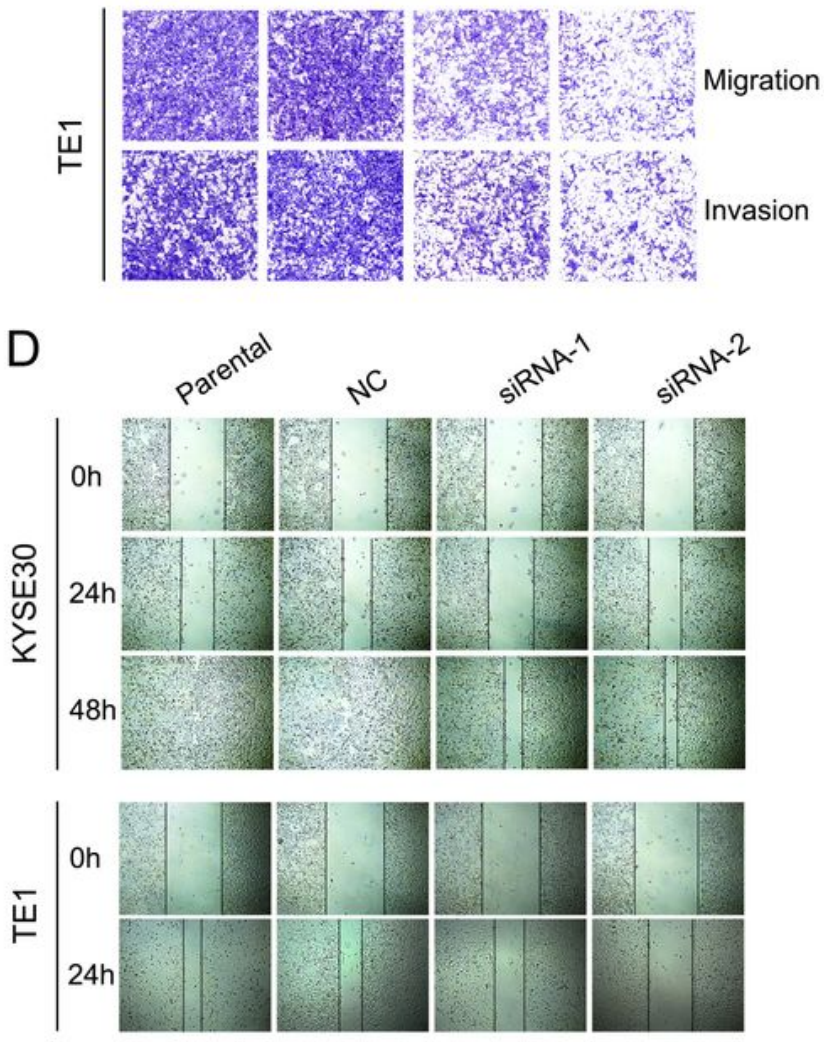

B
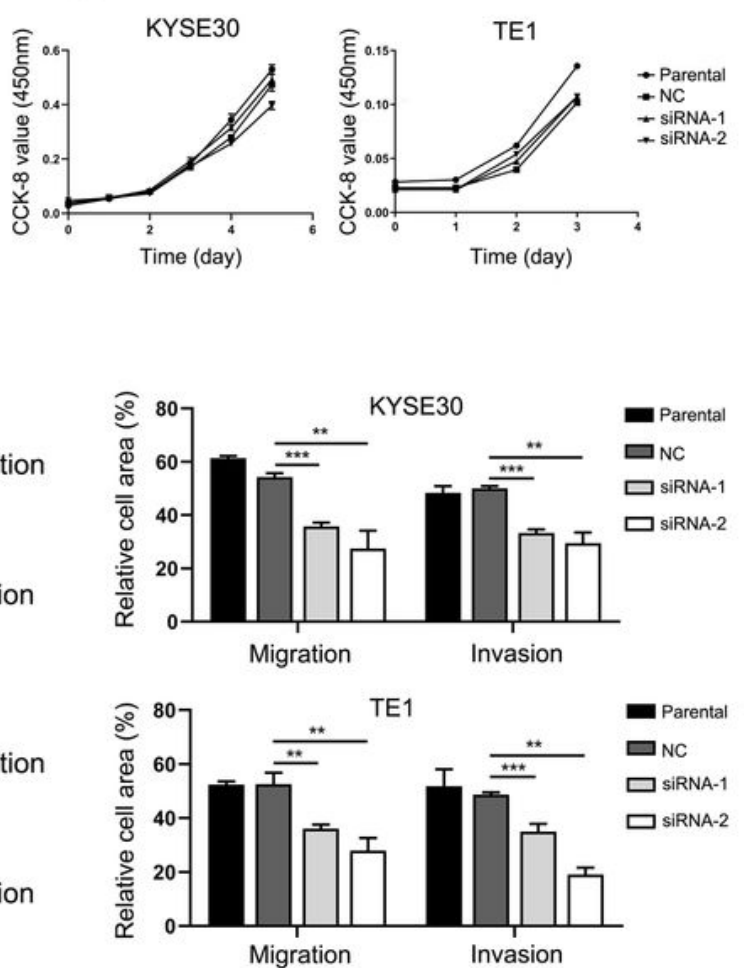

E

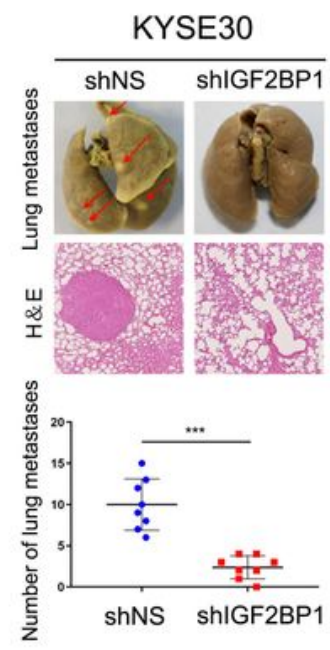

\section{Figure 2}

IGF2BP1 promotes ESCC cells migration, invasion in vitro and lung metastasis in mice. A. Western blot analysis of IGF2BP1 expression in ESCC cell lines B. Cell viability was quantified using CCK-8 assay. C. Cell migration and invasion abilities were examined using transwell assay. Representative results (left) and statistical plots (right) are shown. D. Cell motility was assessed by the wound-healing assay. E. Cell metastatic potential was evaluated using an in vivo pulmonary metastasis assay. Representative images 
of fixed lung tissues (top) and the results of H\&E staining (bottom) are shown. The arrows indicate the lung metastatic nodules. The number of metastatic nodules was plotted (right).

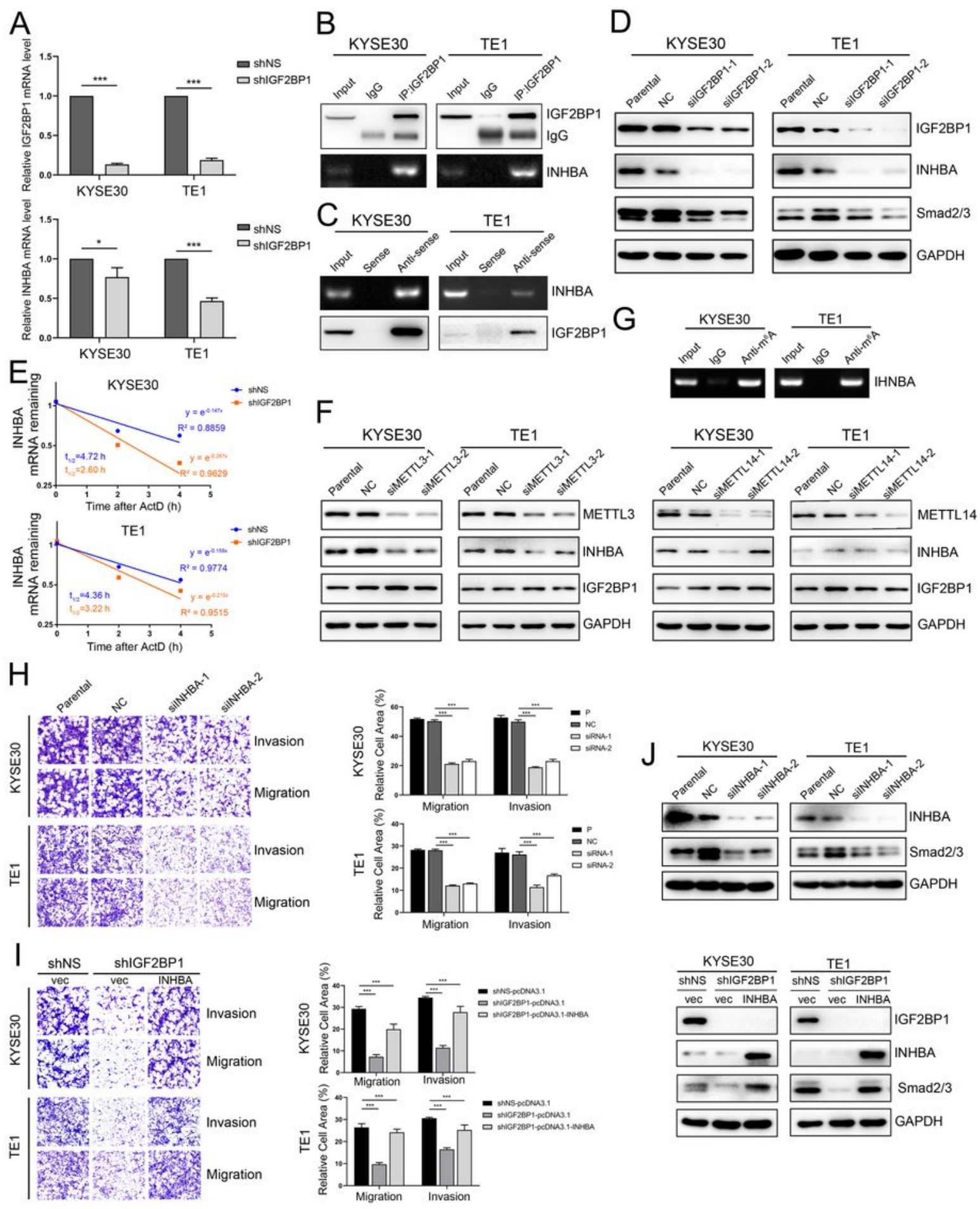

\section{Figure 3}

IGF2BP1 enhances ESCC cells migration and invasion via activating INHBA-Smad2/3 signaling. A-B. Interaction between IGF2BP1 protein and INHBA mRNA in ESCC cells was validated with RIP (A) and RNA pull-down assay (B). IGF2BP1 protein enriched by antibody or co-precipitated by biotin labeled DNA probe 
against INHBA mRNA was detected by using western blotting; INHBA mRNA enriched by biotinylated probe or co-precipitated by IGF2BP1 antibody was analyzed by using RT-PCR. IgG antibody or biotinlabeled sense probe was used as negative control. C. The mRNA level of IGF2BP1 and INHBA in KYSE30 and TE1 cells after IGF2BP1 knockdown were determined by real-time PCR. D. Western blot analysis of IGF2BP1, INHBA, Smad2/3 in ESCC cells transfected with IGF2BP1 specific siRNA or NC siRNA. E. The decay rate of INHBA mRNA after IGF2BP1 depletion was evaluated by RNA stability assay. Relative mRNA expression at 0,2 , $4 \mathrm{~h}$ after ActD treatment was analyzed by real-time PCR, and GAPDH was used as loading control. The decay rate of mRNA was measured in terms of half-life time(t1/2). F. Western blot analysis of IGF2BP1, INHBA, METTL3/14 after METTL3/14 transient knockdown. G. m6A modification in INHBA mRNA was tested by gene-specific m6A PCR. Methylated INHBA mRNA in total RNA enriched by m6A antibody was analyzed by RT-PCR. IgG antibody served as negative control. H-I. Cell migration and invasion abilities examined with transwell assays. Representative results (left) and statistical plots (right) are shown. J. Western blot analysis of IHNBA and Smad2/3 in ESCC cells transfected with INHBA siRNA (above) and in ESCC cells stably expressing shIGF2BP1 transfected with pcDNA3.1-INHBA or empty vector (below). ${ }^{*} \mathrm{P}<0.05 ; * \star \star \mathrm{P}<0.001$. 


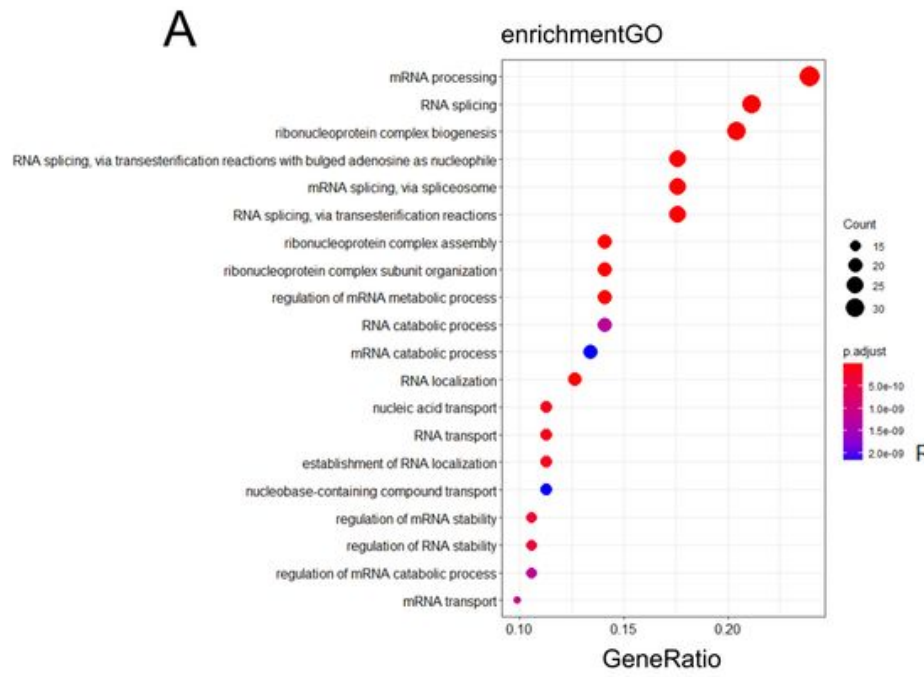

C


B

enrichmentKEGG

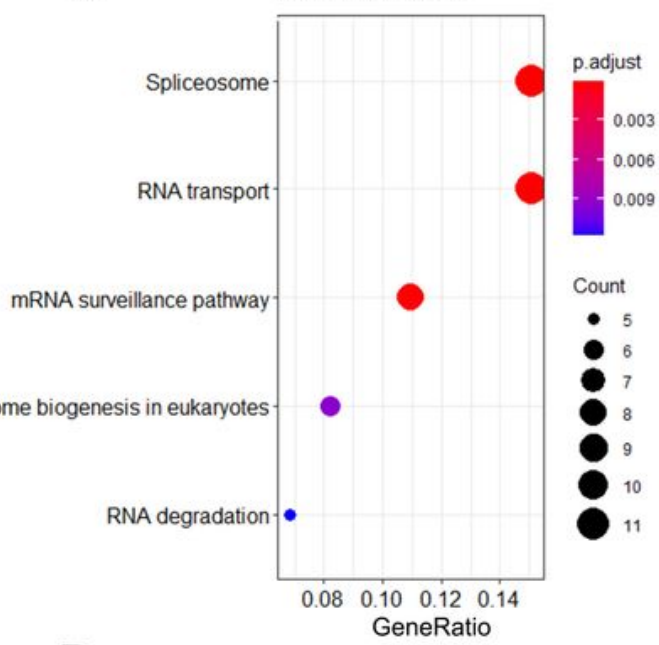

D
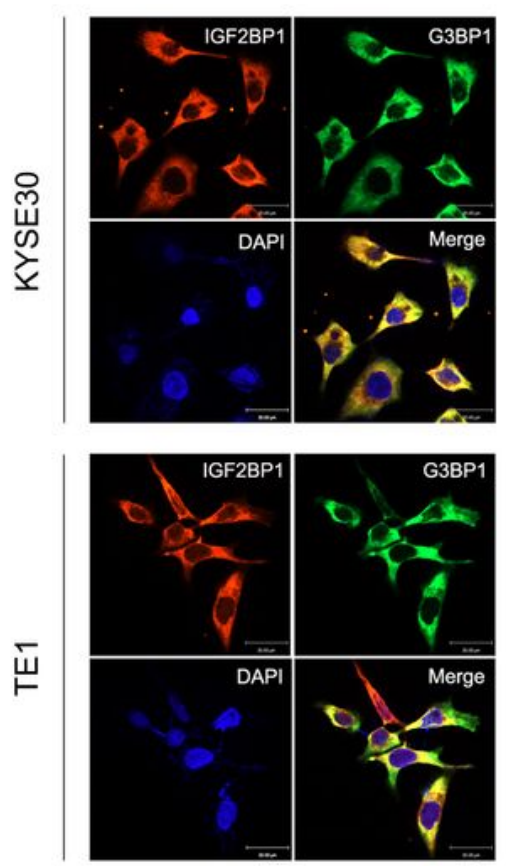

\section{Figure 4}

G3BP1 interacts with IGF2BP1 and facilitates to activate INHBA-Smad2/3 signaling. A-B. Bubble plot of GO (A) and KEGG (B) enrichment based on mass spectrometry results. The alteration in color from red to blue illustrates a decrease in the P-value, and the dot size indicates the number of genes enriched in KEGG terms. C. Interaction between IGF2BP1 and G3BP1 in ESCC cells was detected with an endogenous immunoprecipitation assay. D. Cellular localization of exogenously expressed IGF2BP1 (red) or G3BP1 
(green) was detected by immunofluorescence staining by using laser confocal microscopy, DAPI was used to stain nuclei (blue). Scale bar $=30 \mu \mathrm{M}$. E. Western blot analysis of IGF2BP1, INHBA, Smad2/3 in ESCC cells transfected with NC-siRNA or G3BP1 siRNA. GAPDH was used as loading control.

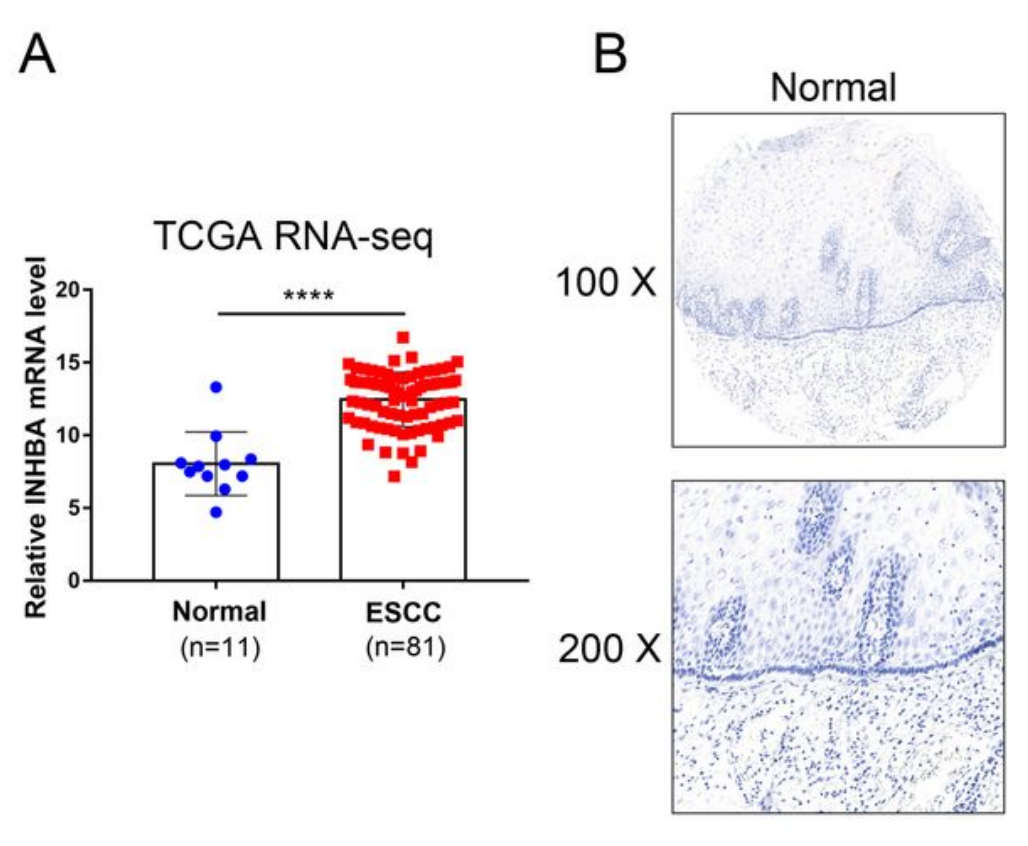

C

Expression of INHBA in

Head and Neck squamous cell carcinoma

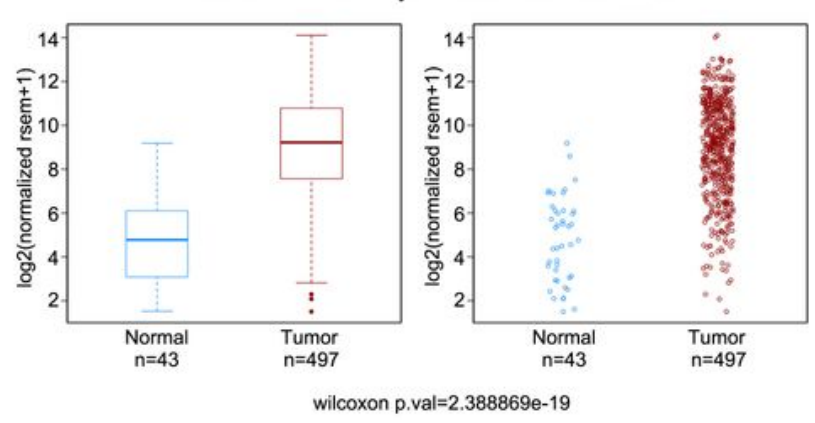

ESCC tissues

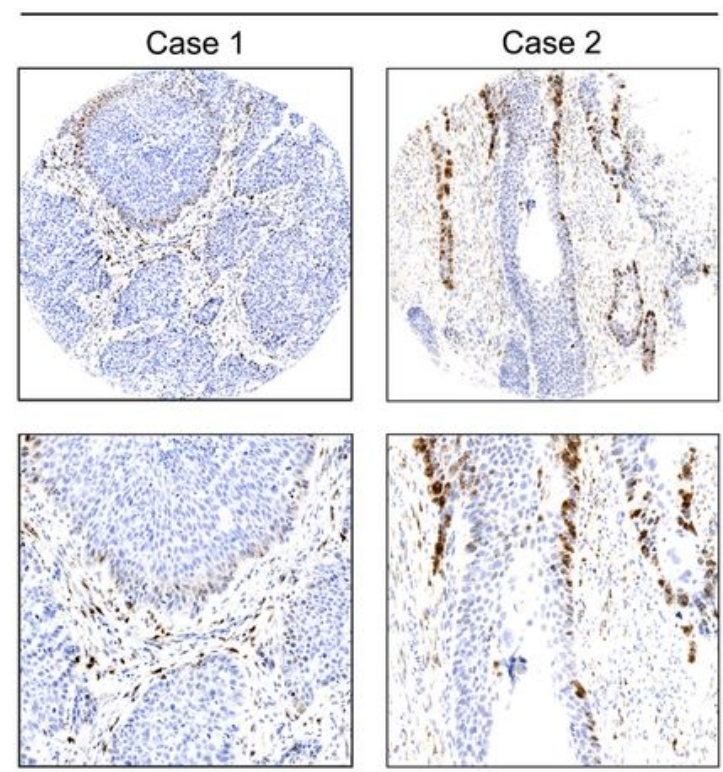

Expression of INHBA in

Breast invasive carcinoma
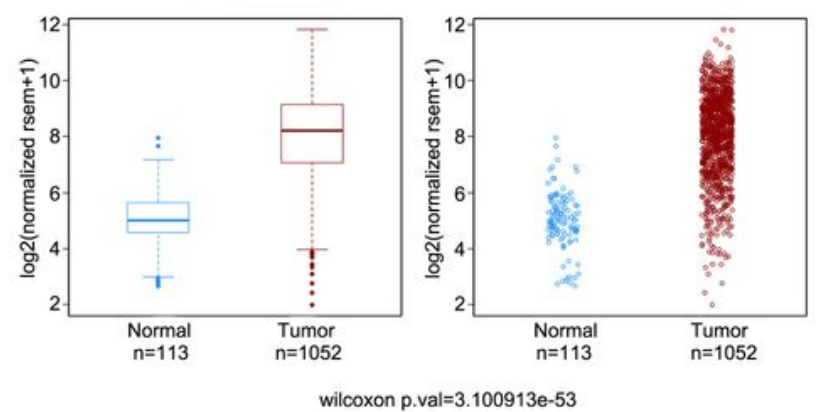

\section{Figure 5}

INHBA is upregulated in ESCC, HNSC and invasive breast cancer. A. INHBA mRNA level in ESCC and normal tissues in TCGA database. ${ }^{* \star * * P}<0.0001$. B. Representative RISH staining of INHBA mRNA in 
ESCC and adjacent normal tissues. C. INHBA mRNA level in HNSC, invasive breast cancer and normal tissues from TCGA database. $P<0.0001$.

\section{A}
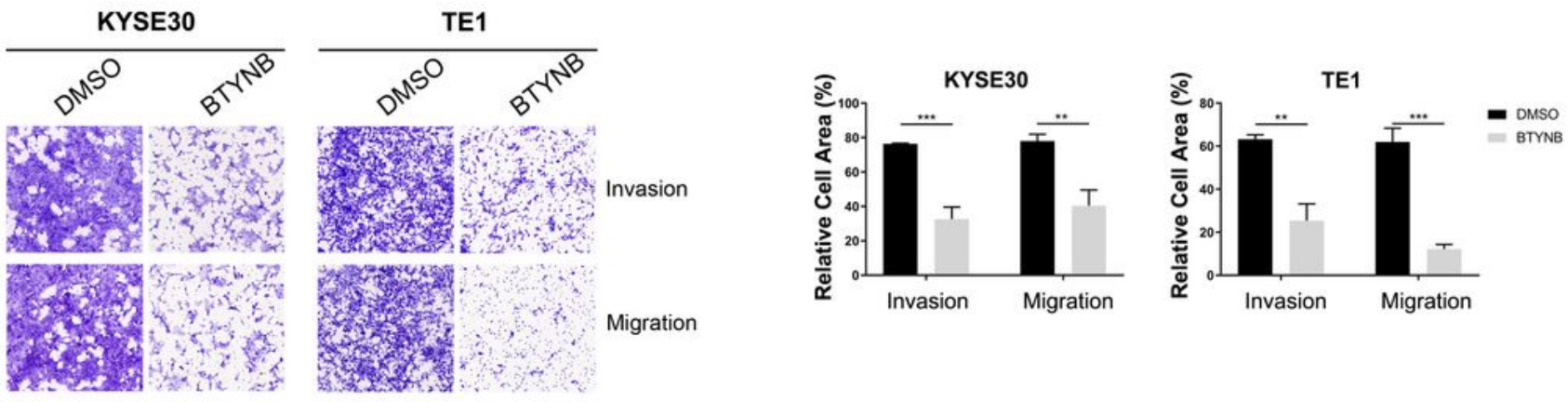

B

BTYNB
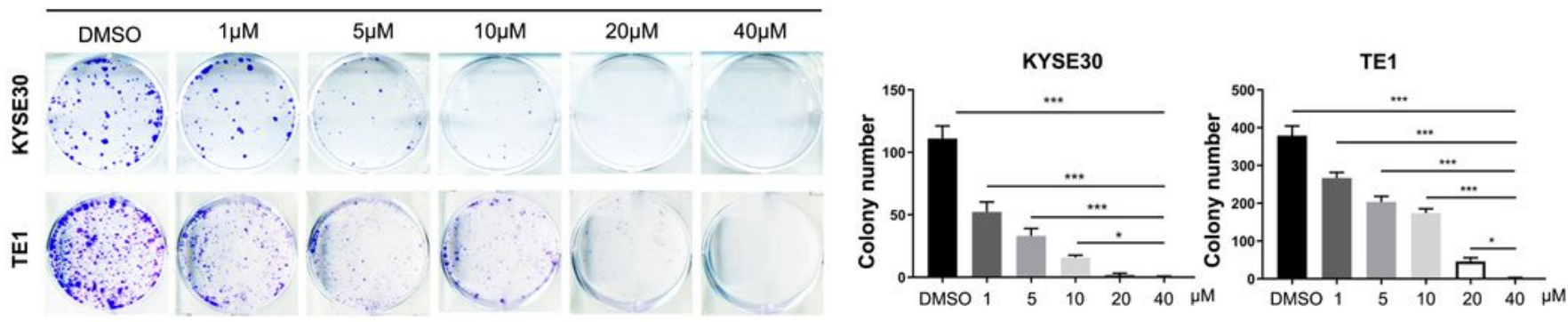

\section{C}

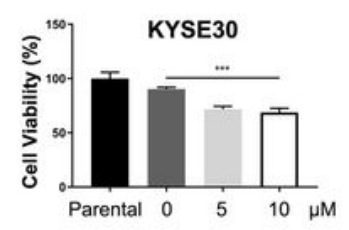

D
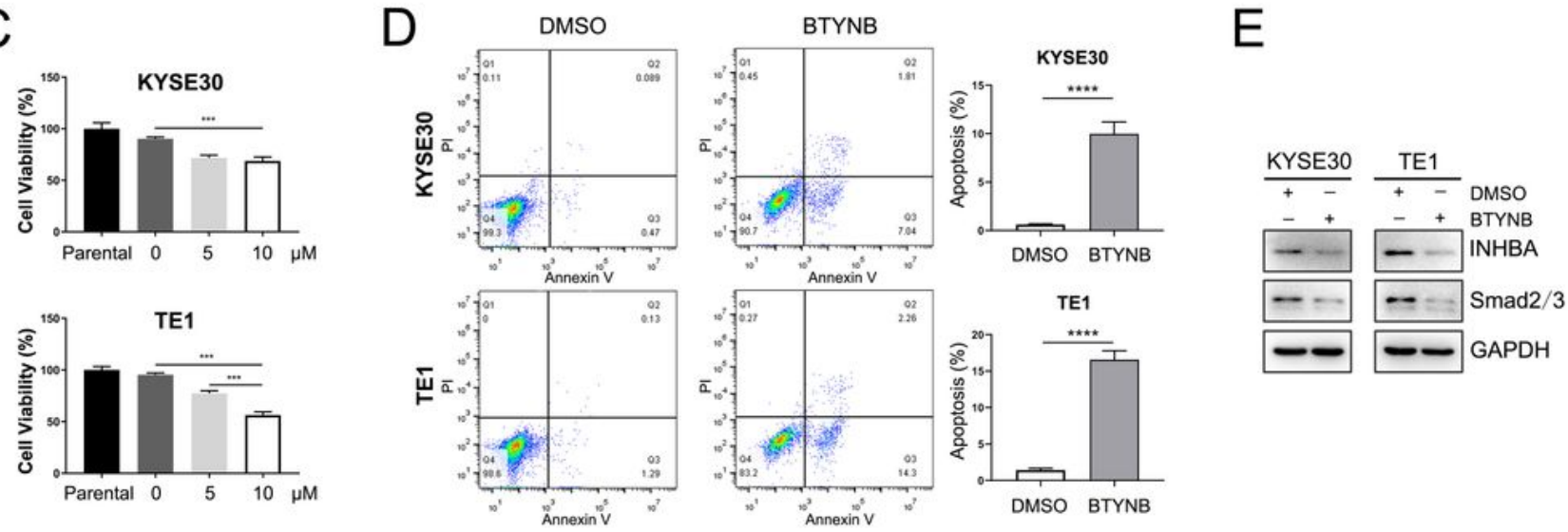

Figure 6

BTYNB inhibits the invasion, migration and proliferation of ESCC cells in vitro. A. The effects of BTYNB on cell migration and invasion were examined by transwell assay. $20 \mu \mathrm{M}$ BTYNB was added to the lower compartment for 24h-36h. Representative images (left)and statistical plots are shown (right). B. Colony 
formation of KYSE30 and TE1 cells exposed to different concentrations of BTYNB. Representative images of indicated groups were shown on the left panel and statistical plots are shown on the right. C. Cell viability of ESCC cells exposed to BTYNB was determined by CCK-8 assay. KYSE30 and TE1 cells were treated with $5 \mu \mathrm{M}$ and $10 \mu \mathrm{M}$ BTYNB for $72 \mathrm{~h}$. D. Cell apoptosis was determined by flow cytometry. Cells were treated with $10 \mu \mathrm{M}$ BTYNB for 48h. Representative images (left)and statistical plots are shown (right). ${ }^{*} P<0.05 ;{ }^{\star *} P<0.01 ; * \star * P<0.001 ; * \star \star \star P<0.001$. E. Western blot analysis of the indicated proteins in cells treated with BTYNB. GAPDH was used as loading control.

A
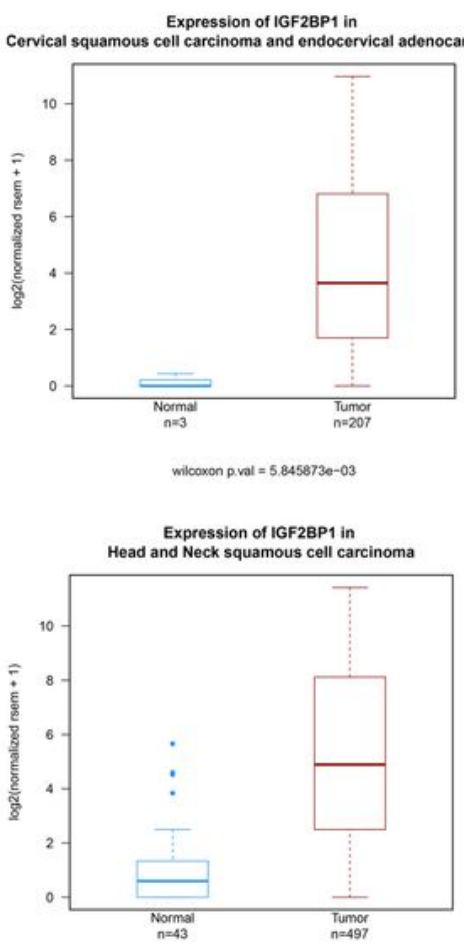

wilcoxon pval = 9.141578 - 19

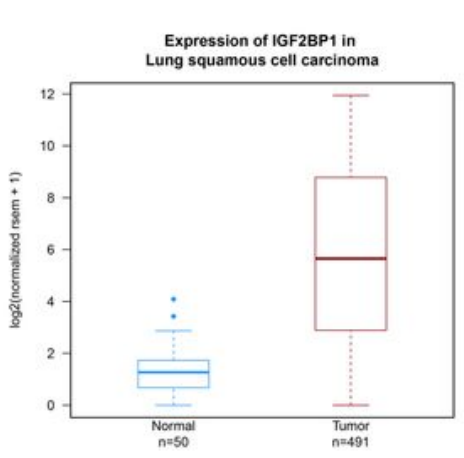

wilcoxon p.val $=4.0556030-20$
B
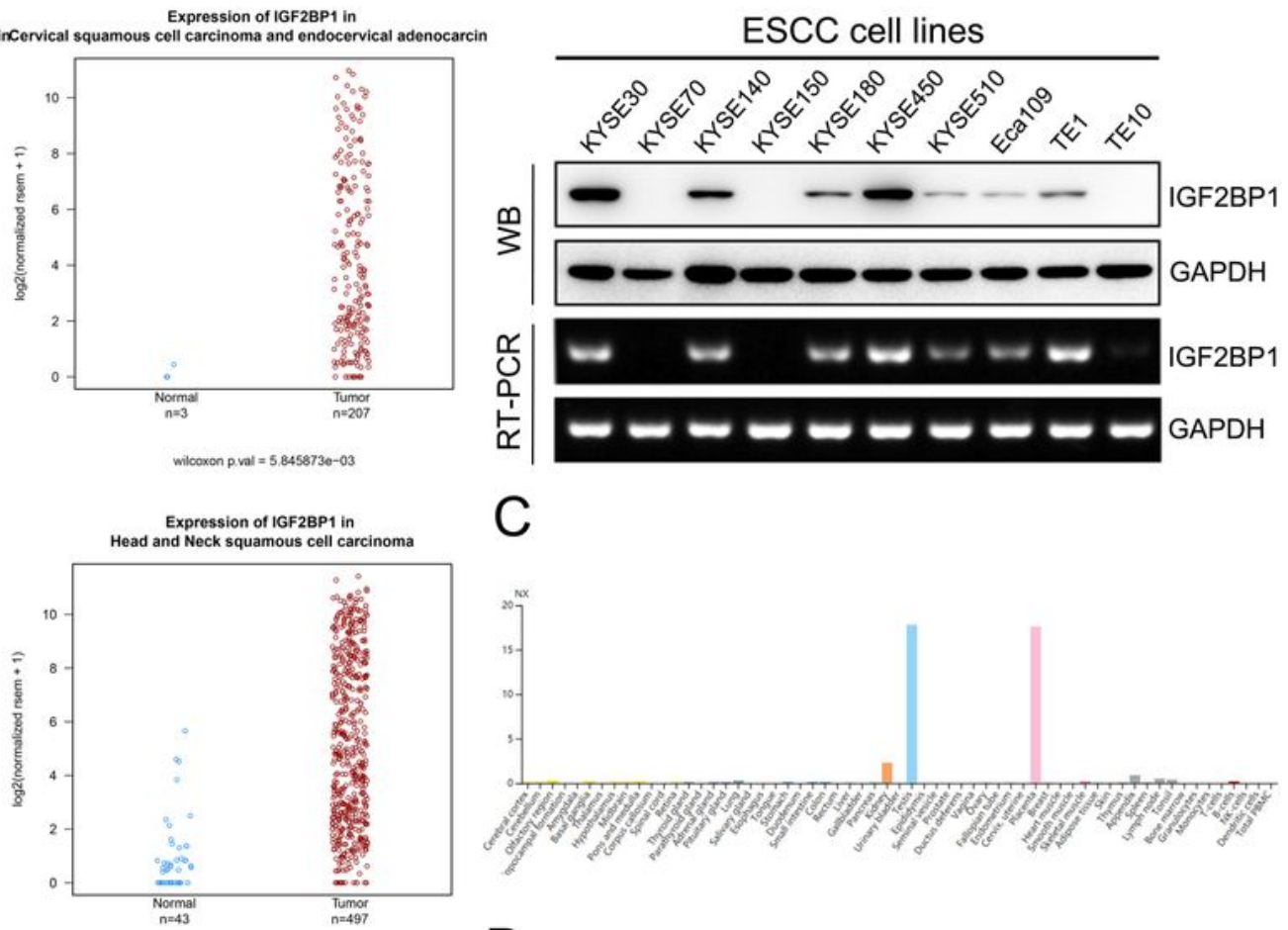

C

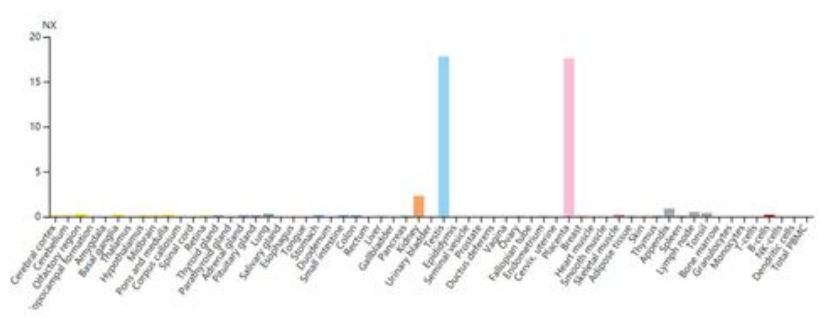

D

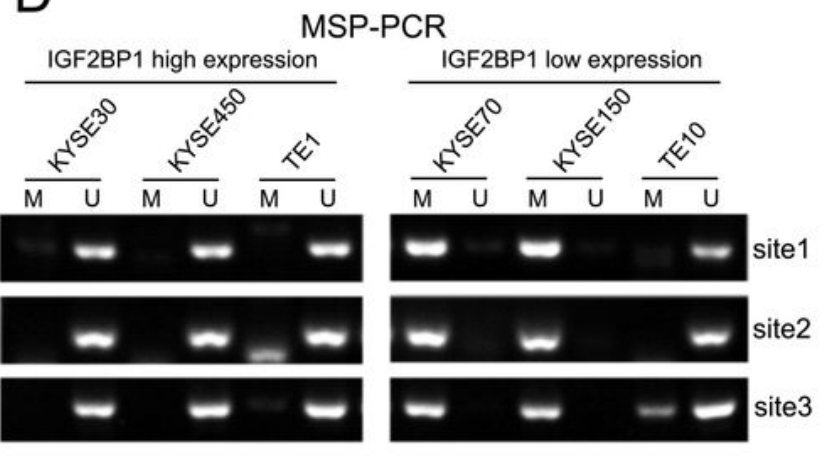




\section{Figure 7}

High IGF2BP1 expression in ESCC cells is associated with genomic hypomethylation. A. IGF2BP1 mRNA level in HNSCC, LUSC, CESC patients and normal tissues in TCGA database. B. IGF2BP1 protein and mRNA levels of ESCC cell lines were analyzed by RT-PCR and western blot, respectively. C. The expression for IGF2BP1 mRNA in all major tissues and organs in the human body was analyzed in HPA database. D. Methylation level of the first intron of IGF2BP1 gene in ESCC cell genome was assessed by MSP-PCR. Genomic DNA was extracted from ESCC cells with high and low IGF2BP1 expression. Three pairs of methylated and unmethylated primers ( $\mathrm{M}$ or $\mathrm{U}$ ) targeting three $\mathrm{CG}$ sites in first intron were designed to amplify DNA converted by bisulfite.

\section{Supplementary Files}

This is a list of supplementary files associated with this preprint. Click to download.

- Additionalfile1.docx

- Additionalfiles2.eps

- Additionalfiles3.eps 